\title{
Effects of Chemical Reaction on MHD Flow Past an Impulsively Started Infinite Vertical Plate with Variable Temperature and Mass Diffusion in the Presence of Hall Current
}

\author{
B. Prabhakar Reddy ${ }^{1,2^{*}}$, J. Peter $^{1}$ \\ ${ }^{1}$ Department of Mathematics, College of Natural \& Mathematical Sciences, \\ The University of Dodoma, P. Box No. 338, Dodoma, Tanzania \\ e-mail: prabhakar.bijjula@gmail.com \\ ${ }^{2}$ Department of Mathematics, Geethanjali College of Engineering \& Technology, \\ Keesara, Medchal - 501301, Telangana, India \\ e-mail:peterjosephat55@gmail.com \\ *corresponding author
}

\begin{abstract}
In this paper, the effects of chemical reaction on unsteady MHD flow of viscous incompressible electrically conducting fluid past an impulsively started oscillating vertical plate with variable temperature and constant mass diffusion in the presence of Hall current have been presented. The dimensionless governing partial differential equations of the flow have been solved numerically by using the Crank-Nicolson implicit finite difference scheme. The numerical solutions for primary fluid velocity, secondary fluid velocity, fluid temperature and fluid concentration are plotted graphically whereas the numerical results of primary skin friction, secondary skin frictions, rate of heat and mass transfer are presented in tabular form for various parameters involved. A comparison has been provided between the present results and with the existing analytical solutions by the Laplace transforms technique. It has been found that the results of the present study are in excellent agreement with previously obtained results.
\end{abstract}

Keywords: MHD, chemical reaction, magnetic parameter, Hall current, oscillating vertical plate

\section{Introduction}

The study of magneto-hydrodynamic (MHD) flow with chemical reaction is of great practical importance to engineers and scientists because of its almost universal occurrence in many branches of science and engineering. In many chemical engineering processes, chemical reaction takes place between a foreign mass and the working fluid in which the plate is moving. These processes take place in numerous industrial applications such as manufacturing of ceramics or glassware, polymer production and food processing. In nature, the presence of pure air or water is not possible. Some foreign mass may be present either naturally or mixed with the air or water. The presence of a foreign mass in air or water causes some kind of chemical reaction. The study of such type of chemical reaction processes is useful in improving a number of chemical technologies, such as food processing and polymer production. Das et al. (1994) studied the effect 
of homogeneous first order chemical reaction on the flow past an impulsively started infinite vertical plate with uniform heat flux and mass transfer. Anjalidevi and Kandasamy (2000) analyzed the effects of chemical reaction on the flow in the presence of heat transfer and magnetic field. Muthucumaraswamy and Ganesan (2001) studied the effect of a chemical reaction on an unsteady flow past an impulsively started vertical plate which is subjected to uniform mass flux and in the presence of heat transfer. Raptis and Perdikis (2006) studied numerically the steady two dimensional flow of an incompressible viscous and electrically conducting fluid over a nonlinear semi infinite stretching sheet in the presence of chemical reaction and under the influence of a magnetic field. Cortell (2007) studied MHD flow and mass transfer of an electrically conducting fluid of second grade over nonlinear stretching sheet with chemically reactive species in a porous medium. Mass transfer effects on isothermal vertical oscillating plate in the presence of chemical reaction were investigated by Muthucumaraswamy and Janakiraman (2008). Mahapatra et al. (2010) analyzed the effects of chemical reaction on free convection flow through a porous medium bounded by a vertical surface. Rajesh and Varma (2010) presented chemical reaction effects on free convection flow past an exponentially accelerated vertical plate. MHD and chemical reaction effects on free convection flow with variable temperature and mass diffusion was reported by Rajesh (2010). El-Fayez (2012) investigated the effects of chemical reaction on unsteady free convection flow past an infinite vertical permeable moving plate with variable temperature. Sehkar and Reddy (2012) presented the effects of chemical reaction on MHD free convective oscillatory flow past a porous plate with viscous dissipation and heat sink.

However, in all these investigations, the effects of Hall current are not taken into account. The effect of Hall current cannot be completely ignored if the strength of the magnetic field is strong and number of density electrons is small as it is responsible for the charge of the flow pattern of an ionized gas. Hall effect results in a development of an additional potential difference between opposite surfaces of a conductor for which a current is induced perpendicular to both the electric and magnetic field. This current is known as Hall current. It plays an important role in determining flow features of the fluid flow problems because it induces secondary flow in the flow field. The problem of MHD viscous flow with Hall current has significant application in several areas of engineering problems such as the development of efficient Hall thrusters in magnetic propulsion (Fife, 1998), plasma actuator control of hypersonic flows (Shang et. al, 2005), influence of Hall effects on the characteristics of MHD generators (Kholshchevnikova, 1966) and super conducting films (Michaeli, 2012), etc. The current movement is towards the application of strong magnetic field i.e. in material processing (Davidson, 1999), MHD energy systems (Hardianto et. al, 2008), MHD sea water propulsion (Mathon, 2009), etc. and towards a low density of the gas i.e., in application of plasma devices for future vehicle systems (Van Wie, 2005), nuclear fission research (Morley et. al, 2005), etc. The effect of Hall current on hydro-magnetic flow near an accelerated plate was presented by Pop (1971). Watanabe and Pop (1995) investigated Hall effect on magneto-hydrodynamic boundary layer flow over a continuous moving flat plate. Aboeldahab and Elbarbury (2001) analyzed the effect of Hall current on magneto-hydrodynamic free convection flow past a semi-infinite vertical plate with mass transfer. Acharya et al. (2001) presented Hall effect with simultaneous thermal and mass diffusion on unsteady hydro-magnetic flow near an accelerated vertical plate. Hall effect on MHD mixed convection flow of a viscous incompressible fluid past a vertical porous plate immersed in a porous medium with heat source/sink was investigated by Sharma et al. (2007). Maguna and Mutua (2013) presented the effects of Hall current on free convection flow and mass transfer past a semi infinite vertical flat plate. Thamizhsudar et al. (2015) analyzed the effects of Hall current and rotation on MHD flow past an exponentially accelerated vertical plate with combined heat and mass transfer effects. MHD flow past a vertical plate with variable temperature and mass diffusion in the presence of Hall current was reported by Rajput and Kanaujia (2016). Recently, Reddy (2018) investigated the effect of Hall current on MHD transient flow past an impulsively started infinite horizontal porous plate in a rotating system. 
The objective of the present study is to investigate the effects of chemical reaction on unsteady MHD flow past an impulsively started oscillating infinite vertical plate with variable temperature and constant mass diffusion in the presence of Hall current. The main purpose of the present study is to extend the recent work of Rajput and Kanaujia (2016) with incorporating the effect of chemical reaction which should increase the applicability of the studies reported in the literature. A comparison of the present results in the absence of chemical reaction was made with the results of Rajput and Kanaujia (2016) obtained by exact solution (Laplace transform technique). It was found that our results are in excellent agreement with the results of Rajput and Kanaujia (2016).

\section{Formulation of the Problem}

Consider the unsteady MHD flow of viscous incompressible electrically conducting fluid past an impulsively started oscillating vertical plate taking chemical reaction into account. A uniform magnetic field $\vec{B}$ of strength $B_{0}$ is applied in the direction perpendicular to the fluid flow. Choose a Cartesian co-ordinate system with the $x^{\prime}$ - axis along the plate in the vertically upward direction, the $y^{\prime}-$ axis perpendicular to the direction of the plate and $z^{\prime}-$ axis is normal to the $x^{\prime} y^{\prime}-$ plane. The physical model of the problem is shown in Fig.1. Initially, at time $t^{\prime} \leq 0$, the temperature of the fluid and the plate is $T_{\infty}^{\prime}$ and the concentration of the fluid is $C_{\infty}^{\prime}$. At time $t^{\prime}>0$, the plate starts oscillating in its own plane with frequency $\omega^{\prime}$, the temperature of the plate and the concentration of the fluid, respectively are raised to $T_{w}^{\prime}$ and $C_{w}^{\prime}$. As the plate is of infinite extent and electrically non-conducting, all the physical quantities, except the pressure, are functions of $y^{\prime}$ and $t^{\prime}$.

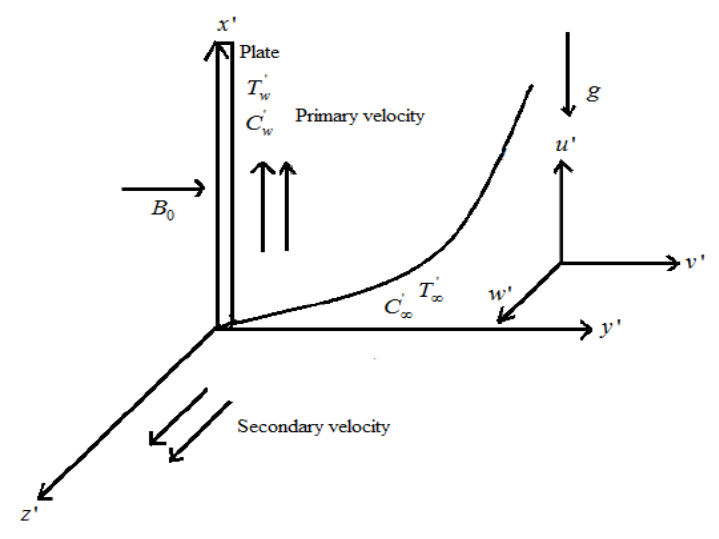

Fig. 1. Flow geometry and physical coordinate system

The generalized Ohm's law on taking Hall current into account Cowling, (1957) is given by:

$$
\vec{J}+\frac{\omega_{e} \tau_{e}}{B_{0}}(\vec{J} \times \vec{B})=\sigma(\vec{B}+\vec{q} \times \vec{B})
$$

where $\vec{q}, \vec{B}, \vec{E}, \vec{J}, \sigma, \omega_{e}$ and $\tau_{e}$ are respectively, velocity vector, magnetic field vector, electric field vector, current density vector, electric conductivity, cyclotron frequency and electron 
collision time. The equation of continuity $\nabla \cdot \vec{q}=0$ gives $v^{\prime}=0$ everywhere in the flow since there is no variation of the flow in $y^{\prime}$ - direction, where $\vec{q}=\left(u^{\prime}, v^{\prime}, w^{\prime}\right)$ and $u^{\prime}, v^{\prime}, w^{\prime}$ are respectively, velocity components along the coordinate axes. The magnetic Reynolds number is so small that the induced magnetic field produced by the fluid motion is neglected. The solenoid relation $\nabla \cdot \vec{B}=0$ for the magnetic field $\vec{B}=\left(B_{x^{\prime}}, B_{y^{\prime}}, B_{z^{\prime}}\right)$ gives $B_{y^{\prime}}=$ constant say $B_{0}$. i.e., $\vec{B}=\left(0, B_{0}, 0\right)$ everywhere in the flow. The conservation of electric current $\nabla \cdot \vec{J}=0$ yields $j_{y^{\prime}}=$ constant, where $\vec{J}=\left(j_{x^{\prime}}, j_{y^{\prime}}, j_{z^{\prime}}\right)$. This constant is zero since $j_{y^{\prime}}=0$ at the plate which is electrically non- conducting. Hence, $j_{y^{\prime}}=0$ everywhere in the flow.

In view of the above assumption, equation (1) yields

$$
\begin{aligned}
& j_{x^{\prime}}-m j_{y^{\prime}}=\sigma\left(E_{x^{\prime}}-w^{\prime} B_{0}\right) \\
& j_{z^{\prime}}+m j_{x^{\prime}}=\sigma\left(E_{z^{\prime}}+u^{\prime} B_{0}\right)
\end{aligned}
$$

where $m\left(=\omega_{e} \tau_{e}\right)$ is the Hall parameter which represents the ratio of electron-cyclotron frequency and the electron-atom collision frequency. Since the induced magnetic field is neglected, Maxwell equation $\nabla \times \vec{E}=-\frac{\partial \vec{H}}{\partial t}$ becomes $\nabla \times \vec{E}=0$ which gives $\frac{\partial E_{x^{\prime}}}{\partial y^{\prime}}=0$ and $\frac{\partial E_{z^{\prime}}}{\partial y^{\prime}}=0$. This implies that $E_{x^{\prime}}=$ constant and $E_{z^{\prime}}=$ constant everywhere in the flow and choose this constants equals to zero, i.e., $E_{x^{\prime}}=E_{z^{\prime}}=0$.

Solving for $j_{x^{\prime}}$ and $j_{z^{\prime}}$ from Eqs. (2) and (3), on using $E_{x^{\prime}}=E_{z^{\prime}}=0$,

$$
\begin{aligned}
& j_{x^{\prime}}=\frac{\sigma B_{0}}{1+m^{2}}\left(m u^{\prime}-w^{\prime}\right) \\
& j_{z^{\prime}}=\frac{\sigma B_{0}}{1+m^{2}}\left(m w^{\prime}+u^{\prime}\right)
\end{aligned}
$$

Taking into consideration the assumptions made above, under the Boussinesq's approximation, i.e., the density changes with temperature, which gives rise to the buoyancy force, and using Eqs. (4) and (5), the basic governing equations of the flow are derived as:

$$
\begin{gathered}
\frac{\partial u^{\prime}}{\partial t^{\prime}}=v \frac{\partial^{2} u^{\prime}}{\partial y^{\prime 2}}-\frac{\sigma B_{0}^{2}}{\rho\left(1+m^{2}\right)}\left(u^{\prime}+m w^{\prime}\right)+g \beta\left(T^{\prime}-T_{\infty}^{\prime}\right)+g \beta^{*}\left(C^{\prime}-C_{\infty}^{\prime}\right) \\
\frac{\partial w^{\prime}}{\partial t^{\prime}}=v \frac{\partial^{2} w^{\prime}}{\partial y^{\prime 2}}+\frac{\sigma B_{0}^{2}}{\rho\left(1+m^{2}\right)}\left(m u^{\prime}-w^{\prime}\right) \\
\frac{\partial T^{\prime}}{\partial t^{\prime}}=\frac{k}{\rho C_{p}} \frac{\partial^{2} T^{\prime}}{\partial y^{\prime 2}}
\end{gathered}
$$




$$
\frac{\partial C^{\prime}}{\partial t^{\prime}}=D \frac{\partial^{2} C^{\prime}}{\partial y^{\prime 2}}-\gamma^{\prime}\left(C^{\prime}-C_{\infty}^{\prime}\right)
$$

The corresponding initial and boundary conditions are:

$$
\begin{aligned}
& t^{\prime} \leq 0 ; u^{\prime}=0, w^{\prime}=0, T^{\prime}=T_{\infty}{ }^{\prime}, C^{\prime}=C_{\infty}{ }^{\prime} \text { for all } y^{\prime} \\
& t^{\prime}>0 ; u^{\prime}=u_{0} \cos \omega^{\prime} t^{\prime}, w^{\prime}=0, T^{\prime}=T_{\infty}{ }^{\prime}+\left(T_{w}{ }^{\prime}-T_{\infty}{ }^{\prime}\right), C^{\prime}=C_{w}{ }^{\prime} \text { at } y^{\prime}=0 \\
& u^{\prime} \rightarrow 0, w^{\prime} \rightarrow 0, T^{\prime} \rightarrow T_{\infty}{ }^{\prime}, C^{\prime} \rightarrow C_{\infty}{ }^{\prime} \text { as } y^{\prime} \rightarrow \infty
\end{aligned}
$$

where $u^{\prime}, w^{\prime}, g, \beta, \beta^{*}, t^{\prime}, C^{\prime}, C_{\infty}^{\prime}, C_{w}^{\prime}, D, T^{\prime}, T_{\infty}^{\prime}, T_{w}^{\prime}, k, v, \rho$ and $C_{p}$ are respectively, velocity of the fluid in $x^{\prime}$ - direction, velocity of the fluid in $z^{\prime}$ - direction, acceleration due gravity, volumetric coefficient of thermal expansion, volumetric coefficient of concentration expansion, time, species concentration in the fluid, concentration in the fluid far away from the plate, species concentration at the plate, mass diffusion, temperature of the fluid, temperature of the fluid near the plate, temperature at the plate, thermal conductivity, kinematic viscosity, fluid density and specific heat at constant pressure.

Let us introduce the following non-dimensional parameters and quantities:

$$
\begin{aligned}
& u=\frac{u^{\prime}}{u_{0}}, y=\frac{y^{\prime} u_{0}}{v}, t=\frac{t^{\prime} u_{0}^{2}}{v}, w=\frac{w^{\prime}}{u_{0}}, \omega=\frac{\omega^{\prime} v}{u_{0}^{2}}, S_{c}=\frac{v}{D}, P_{r}=\frac{\mu C_{p}}{k}, \\
& M=\frac{\sigma B_{0}{ }^{2} v}{\rho u_{0}^{2}}, \gamma=\frac{\gamma^{\prime} v}{u_{0}^{2}}, \theta=\frac{\left(T^{\prime}-T_{\infty}{ }^{\prime}\right)}{\left(T_{w}{ }^{\prime}-T_{\infty}{ }^{\prime}\right)}, \phi=\frac{\left(C^{\prime}-C_{\infty}{ }^{\prime}\right)}{\left(C_{w}{ }^{\prime}-C_{\infty}{ }^{\prime}\right)}, G_{r}=\frac{g \beta v\left(T_{w}{ }^{\prime}-T_{\infty}{ }^{\prime}\right)}{u_{0}{ }^{3}}, \\
& G_{m}=\frac{g \beta v\left(C_{w}^{\prime}-C_{\infty}^{\prime}\right)}{u_{0}^{3}} .
\end{aligned}
$$

Using Eq. (11), into Eqs. (6)-(9), the following dimensionless governing equations of the flow are obtained:

$$
\begin{gathered}
\frac{\partial u}{\partial t}=\frac{\partial^{2} u}{\partial y^{2}}-\frac{M}{\left(1+m^{2}\right)}(u+m w)+G_{r} \theta+G_{m} \phi \\
\frac{\partial w}{\partial t}=\frac{\partial^{2} w}{\partial y^{2}}+\frac{M}{\left(1+m^{2}\right)}(m u-w) \\
\frac{\partial \theta}{\partial t}=\frac{1}{P_{r}} \frac{\partial^{2} \theta}{\partial y^{2}} \\
\frac{\partial \phi}{\partial t}=\frac{1}{S_{c}} \frac{\partial^{2} \phi}{\partial y^{2}}-\gamma \phi
\end{gathered}
$$

where $u, w, \theta, \phi, G_{r}, G_{m}, M, m, P_{r}, S_{c}$ and $\gamma$ are respectively, dimensionless primary velocity, dimensionless secondary velocity, dimensionless temperature, dimensionless concentration, thermal Grashof number, mass Grashof number, magnetic parameter, Hall parameter, Prandtl number, Schmidt number and chemical reaction parameter.

The initial and boundary conditions (10), in non-dimensional form become: 


$$
\begin{aligned}
& t \leq 0 ; u=0, w=0, \theta=0, C=0 \quad \forall y \\
& t>0 ; u=\cos \omega t, w=0, \theta=t, C=1 \text { at } y=0 \\
& u \rightarrow 0, w \rightarrow 0, \theta \rightarrow 0, C \rightarrow 0 \text { as } y \rightarrow \infty
\end{aligned}
$$

\section{Method of solution}

The above system of governing partial differential equations (12)-(15), subject to initial and boundary conditions (16) have been solved numerically for various values of parameters of interest by using the well known Crank-Nicolson implicit finite difference scheme. The finite difference equations corresponding to the equations (12)-(15) are as follows:

$$
\begin{gathered}
\frac{u_{i, j+1}-u_{i, j}}{\Delta t}=\frac{u_{i-1, j+1}-2 u_{i, j+1}+u_{i+1, j+1}+u_{i-1, j}-2 u_{i, j}+u_{i+1, j}}{2(\Delta y)^{2}} \\
+G_{r} \theta_{i, j}+G_{m} \phi_{i, j}-\frac{M}{\left(1+m^{2}\right)}\left(u_{i, j}+m w_{i, j}\right) \\
\frac{w_{i, j+1}-w_{i, j}}{\Delta t}=\frac{w_{i-1, j+1}-2 w_{i, j+1}+w_{i+1, j+1}+w_{i-1, j}-2 w_{i, j}+w_{i+1, j}}{2(\Delta y)^{2}} \\
-\frac{M}{\left(1+m^{2}\right)}\left(w_{i, j}-m u_{i, j}\right) \\
\frac{\theta_{i, j+1}-\theta_{i, j}}{\Delta t}=\frac{1}{P_{r}} \frac{\theta_{i-1, j+1}-2 \theta_{i, j+1}+\theta_{i+1, j+1}+\theta_{i-1, j}-2 \theta_{i, j}+\theta_{i+1, j}}{2(\Delta y)^{2}} \\
\frac{\phi_{i, j+1}-\phi_{i, j}}{\Delta t}=\frac{1}{S_{c}} \frac{\phi_{i-1, j+1}-2 \phi_{i, j+1}+\phi_{i+1, j+1}+\phi_{i-1, j}-2 \phi_{i, j}+\phi_{i+1, j}}{2(\Delta y)^{2}}-\gamma \phi_{i, j}
\end{gathered}
$$

The initial and boundary conditions (16)become:

$$
\begin{aligned}
& u_{i, 0}=0, w_{i, 0}=0, \theta_{i, 0}=0, \phi_{i, 0}=0 \\
& u_{0, j}=\cos (\omega t), w_{0, j}=0, \theta_{0, j}=t, \phi_{0, j}=1 \\
& u_{\max , j}=0, w_{\max , j}=0, \theta_{\max , j}=0, \phi_{\max , j}=0
\end{aligned}
$$

Here the index $i$ refers to $y$ and $j$ refers to time $t$, also $\Delta y=y_{i+1}-y_{i}$ and $\Delta t=t_{i+1}-t_{i}$. The flow domain is restricted to a rectangle of finite dimensions with $y \rightarrow \infty$ is approximated by $y_{\max }=6$ and $t_{\max }=4$, where $\Delta y=0.025$ and $\Delta t=0.0025$ are mesh sizes such that the computational domain is divided into $240 \times 1600$ mesh points. These step sizes are finalized when the code was run with slightly changed values of mesh sizes, and no significant change was observed. This is justified since the boundary condition (21) is satisfied within the tolerance of $10^{-4}$. The finite difference equations at each $j^{\text {th }}$ level constitute a tri-diagonal system of equations which were solved by using Thomas algorithm. During the numerical computation it was found that absolute difference between the numerical values of fluid velocity, fluid temperature and fluid concentration for two consecutive time steps is less than 0.00001 . Hence, the scheme designed is stable. Also, the Crank-Nicolson method has a local truncation error of 
$O\left\{(\Delta y)^{2}+(\Delta t)^{2}\right\}$ which tends to zero as $\Delta y \rightarrow 0$ and $\Delta t \rightarrow 0$ which justifies the consistency of the scheme. Stability and consistency together ensure convergence of the scheme.

The primary skin friction $\tau_{x}$ and secondary skin friction $\tau_{z}$ are given by

$$
\tau_{x}=\left.\frac{\partial u}{\partial y}\right|_{y=0} \text { and } \tau_{z}=\left.\frac{\partial w}{\partial y}\right|_{y=0}
$$

The Nusselt number $N u$ and Sherwood number $S h$ are given by

$$
N_{u}=-\left.\frac{\partial \theta}{\partial y}\right|_{y=0} \text { and } S h=-\left.\frac{\partial \phi}{\partial y}\right|_{y=0}
$$

Validation of the scheme: To validate the present numerical scheme, a comparison has been made by considering primary and secondary skin frictions $\tau_{x}$ and $\tau_{z}$ in the absence of chemical reaction with the results of Rajput and Kanaujia (2016) obtained by analytical solution (Laplace transform technique) shown in Table 1 and 2. An excellent agreement is noticed in this comparison which validates the present numerical scheme. This justifies the correctness of the results presented in the manuscript.

\begin{tabular}{|c|c|c|c|c|c|c|c|}
\hline$G_{r}$ & $G_{m}$ & $M$ & $m$ & $\begin{array}{c}\text { Presented } \\
\text { result } \\
\tau_{x}\end{array}$ & $\begin{array}{c}\text { Results by } \\
\text { Rajput and } \\
\text { Kanaujia } \\
(2016) \\
\tau_{x}\end{array}$ & $\begin{array}{c}\text { Presented } \\
\text { result } \\
\tau_{z}\end{array}$ & $\begin{array}{c}\text { Results by } \\
\text { Rajput and } \\
\text { Kanaujia } \\
(2016) \\
\tau_{z}\end{array}$ \\
\hline 10.0 & 10.0 & 2.0 & 0.5 & 1.1780 & 1.1778 & 0.2149 & 0.2150 \\
\hline 20.0 & 10.0 & 2.0 & 0.5 & 1.5309 & 1.5307 & 0.2211 & 0.2208 \\
\hline 10.0 & 20.0 & 2.0 & 0.5 & 3.1769 & 3.1768 & 0.2566 & 0.2560 \\
\hline 10.0 & 10.0 & 3.0 & 0.5 & 0.9621 & 0.9621 & 0.3065 & 0.3062 \\
\hline 10.0 & 10.0 & 2.0 & 1.0 & 1.3388 & 1.3387 & 0.2799 & 0.2795 \\
\hline
\end{tabular}

Table 1. Primary and secondary skin frictions for $P_{r}=0.71, S_{c}=2.01, \omega t=30^{\circ}, t=0.2$ when

$$
\gamma=0
$$

\begin{tabular}{|c|c|c|c|c|c|c|c|}
\hline$P_{r}$ & $S_{c}$ & $\omega t$ & $t$ & $\begin{array}{c}\text { Presented } \\
\text { result } \\
\tau_{x}\end{array}$ & $\begin{array}{c}\text { Results by } \\
\text { Rajput and } \\
\text { Kanaujia } \\
(2016) \\
\tau_{x}\end{array}$ & $\begin{array}{c}\text { Presented } \\
\text { result } \\
\tau_{z}\end{array}$ & $\begin{array}{c}\text { Results by } \\
\text { Rajput and } \\
\text { Kanaujia } \\
(2016) \\
\tau_{z}\end{array}$ \\
\hline 0.71 & 2.01 & 30 & 0.2 & 1.1780 & 1.1178 & 0.2149 & 0.2150 \\
\hline 7.00 & 2.01 & 30 & 0.2 & 1.0070 & 1.0063 & 0.2109 & 0.2107 \\
\hline 0.71 & 5.00 & 30 & 0.2 & 0.6888 & 0.6887 & 0.1977 & 0.1972 \\
\hline 0.71 & 2.01 & 45 & 0.2 & 1.7317 & 1.7314 & 0.1981 & 0.1983 \\
\hline 0.71 & 2.01 & 30 & 0.25 & 1.5748 & 1.5744 & 0.2490 & 0.2489 \\
\hline
\end{tabular}

Table 2. Primary and secondary skin frictions for $G_{r}=10.0, G_{m}=10.0, M=2.0, m=0.5$ when $\gamma=0$. 
As seen from Tables 1 and 2, both primary and secondary skin frictions are enhanced when thermal Grashof number, mass Grashof number, Hall parameter and time increased whereas both primary and secondary skin frictions are reduced when Prandtl number and Schmidt number increased. An increase in the magnetic parameter tends to decrease primary skin friction and increase the secondary skin friction whereas phase angle has the reverse effect.

\section{Results and Discussion}

In order to determine the impact of physical parameters on the flow, numerical calculations have been carried out for different values of $M, m, G_{r}, G_{m}, P_{r}, S_{c}, \gamma, \omega t$ and $t$. The obtained results for primary fluid velocity $u$, secondary fluid velocity $w$, fluid temperature $\theta$ and concentration $\phi$ are presented graphically in Figs. 2-15, whereas numerical values of primary skin friction $\tau_{x}$, secondary skin frictions $\tau_{z}$ and rate of heat and mass transfer are presented in Tables 1-4. Throughout the study, Prandtl number is kept constant at 0.7, which corresponds to air at $293 \mathrm{~K}$ and one atmosphere and Schmidt number is taken 0.23, which corresponds to hydrogen. The default values of other physical parameters are $G_{r}=10, G_{m}=10, M=1, \quad m=0.5, \omega t=30^{\circ}$, $\gamma=1.0$ and time $t=0.2$, unless otherwise specified.

Figure 2 displays the effect of magnetic parameter $M$ on the primary and secondary fluid velocities. The application of a magnetic field perpendicular to the flow direction of an electrically conducting fluid, experiences an electric field and produces current perpendicular to both magnetic field and flow direction. The product of electric field and magnetic field creates a force which is known as Lorentz force. The direction of the Lorentz force is always opposite to the direction of the flow in the absence of applied electric field which opposes the fluid velocity. This implies that increase in the magnetic field parameter tends to retard the primary fluid velocity whereas it tends to accelerate the secondary fluid velocity. This tendency of the magnetic field is clearly supported by the physical reality.
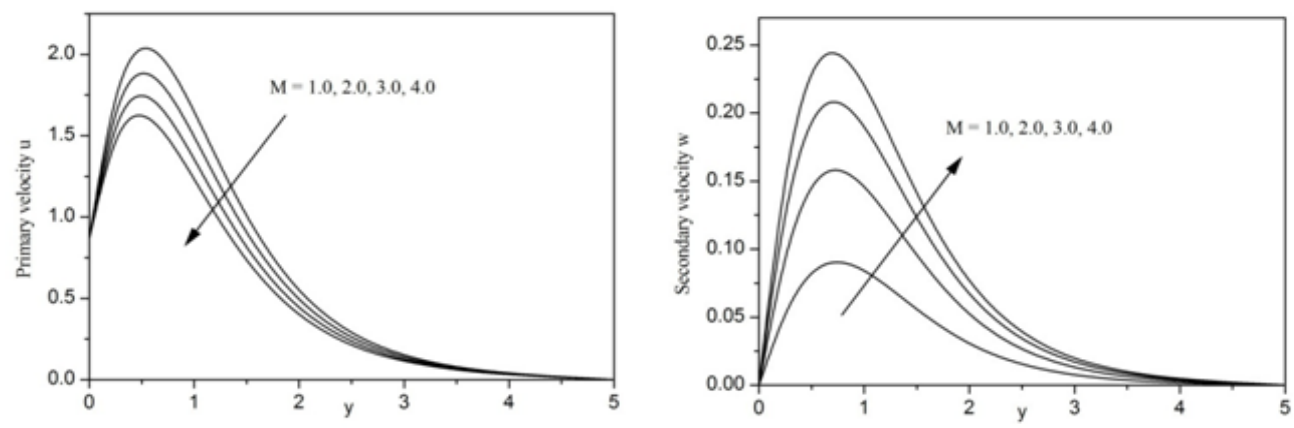

Fig. 2. Primary and secondary fluid velocities for varying magnetic parameter $M$.

The effects of the Hall parameter $m$ on the primary and secondary fluid velocities are depicted in Fig. 3. It is seen that an increase in the Hall parameter tends to accelerate both primary and secondary fluid velocities. This situation supports the fact that the Hall current induces a crossflow in the boundary layer. Figure 4 illustrates the effect of Prandtl number $P_{r}$ on the primary 
and secondary fluid velocities. Prandtl number is the ratio of momentum to thermal diffusivities. Increase in the Prandtl number corresponds to stronger momentum diffusivity and weaker thermal diffusivity. This implies that increase in the Prandtl number tends to decelerate both primary and secondary fluid velocities. Figure 5 shows the influence of Schmidt number $S_{c}$ on the primary and secondary fluid velocities. The values of Schmidt number are chosen as $S_{c}=0.23,0.64,1.20$ and 2.63, which corresponds to hydrogen, water vapor, sulfur dioxide and naphthalene, respectively. Schmidt number is the ratio between viscous forces and mass diffusivity. As $S_{c}$ increases both primary and secondary velocities are expected to reduce since the increase in $S_{c}$ means increase of viscous force which leads the decrease of the velocity components.
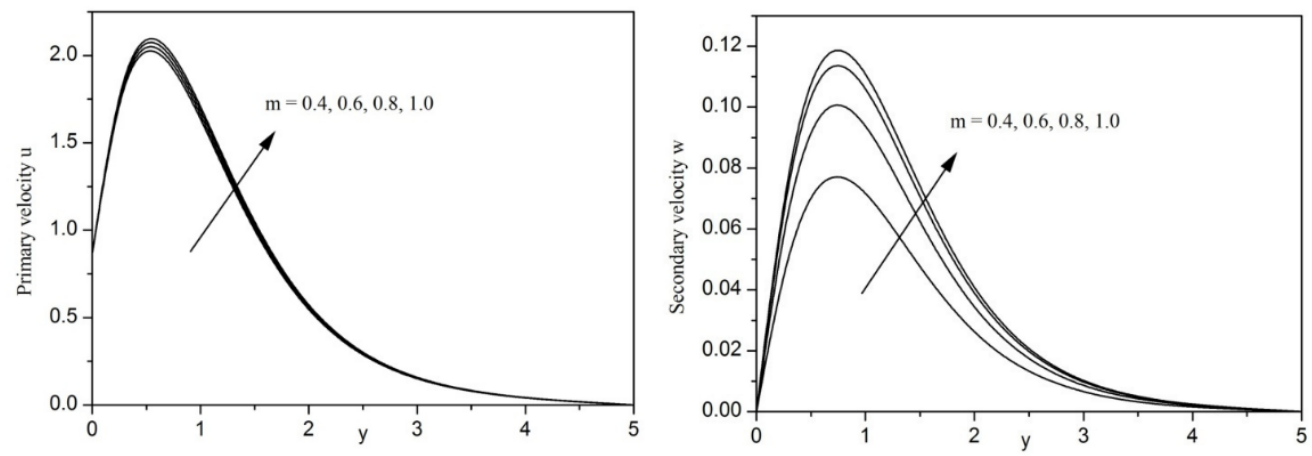

Fig. 3. Primary and secondary fluid velocities for varying Hall parameter $m$.
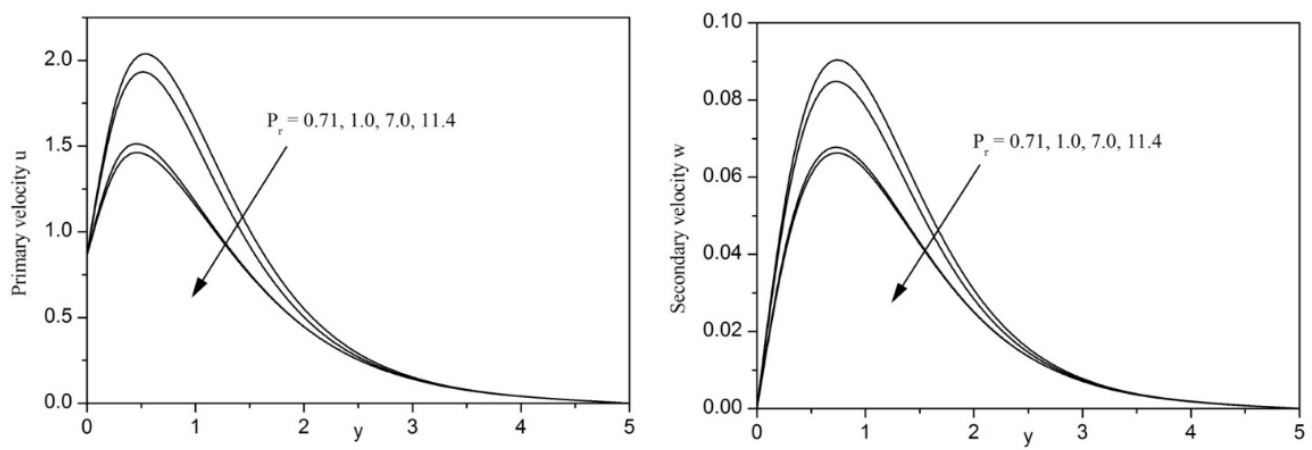

Fig. 4. Primary and secondary fluid velocities for varying Prandtl number $P_{r}$. 

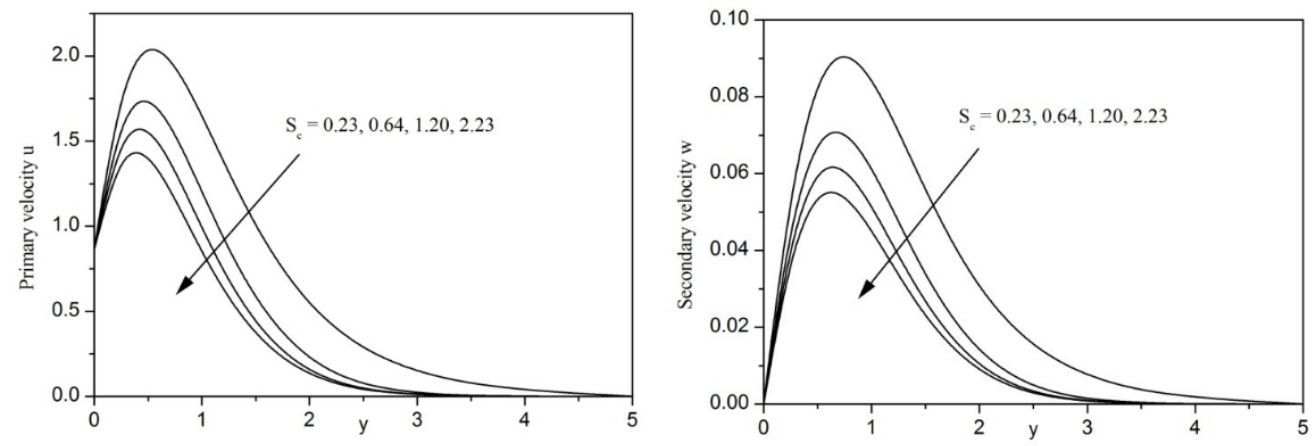

Fig. 5. Primary and secondary fluid velocities for varying Schmidt number $S_{c}$.

The effects of thermal Grashof number $G_{r}$ on the primary and secondary fluid velocities are presented in Fig. 6. As increase in thermal Grashof number $G_{r}$ leads to decrease drag forces and, hence, fluid velocity increase. This implies that the increase in the thermal Grashof number tends to enhance both primary and secondary fluid velocities. Here, the positive values of thermal Grashof number correspond to cooling of the plate. Figure 7 presents the effects of mass Grashof number $G_{m}$ on the primary and secondary fluid velocities. The mass Grashof number defines the ratio of species buoyancy force to the viscous hydrodynamic force. As mass Grashof number increases, the viscous hydrodynamic force decreases. As a result, momentum of the fluid is higher. This implies that both primary and secondary fluid velocities tend to increase with increasing mass Grashof number. Also, it is noticed that both primary and secondary velocities suddenly raise near to the plate and after reaching maximum velocity both velocity components gradually decrease to zero as $y \rightarrow \infty$.
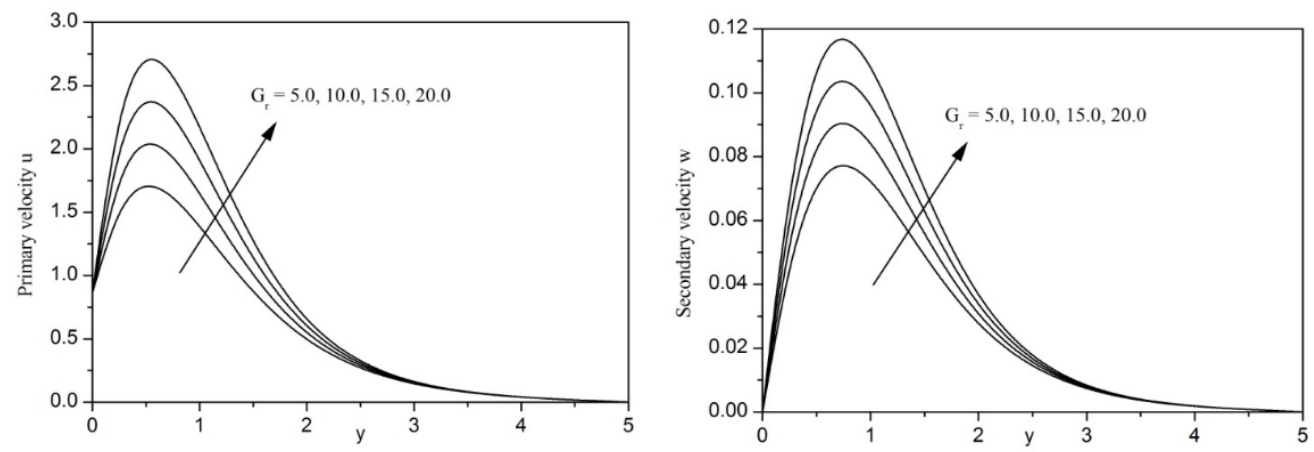

Fig. 6. Primary and secondary fluid velocities for varying thermal Grashof number $G_{r}$. 

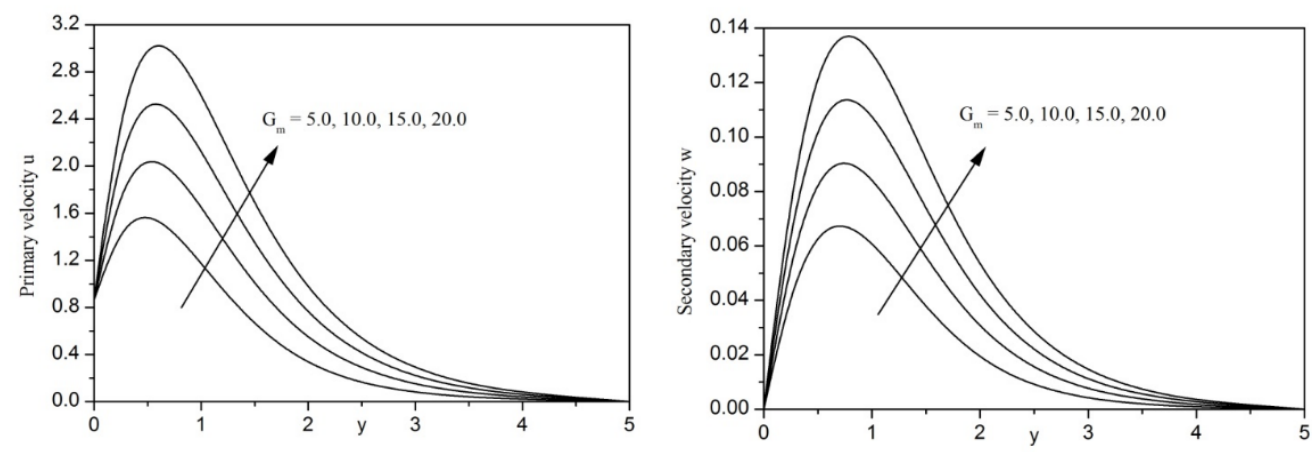

Fig. 7. Primary and secondary fluid velocities for varying mass Grashof number $G_{m}$.

The effects of chemical reaction parameter $\gamma$ on the primary and secondary fluid velocities are depicted in Fig. 8. It can be seen that an increase in $\gamma$ tends to decrease both primary and secondary velocities. Figure 9 shows the effects of phase angle $\omega t$ on the primary and secondary fluid velocities. An increase in the phase angle $\omega t$ leads to decrease the buoyancy force and, hence, decreases the fluid momentum. This implies that increase in the phase angle tends to decelerate both primary and secondary fluid velocities. The effects of time $t$ on the primary and secondary fluid velocities are presented Fig. 10. As time progresses, both primary and secondary velocities are getting accelerated due to increasing buoyancy effects.
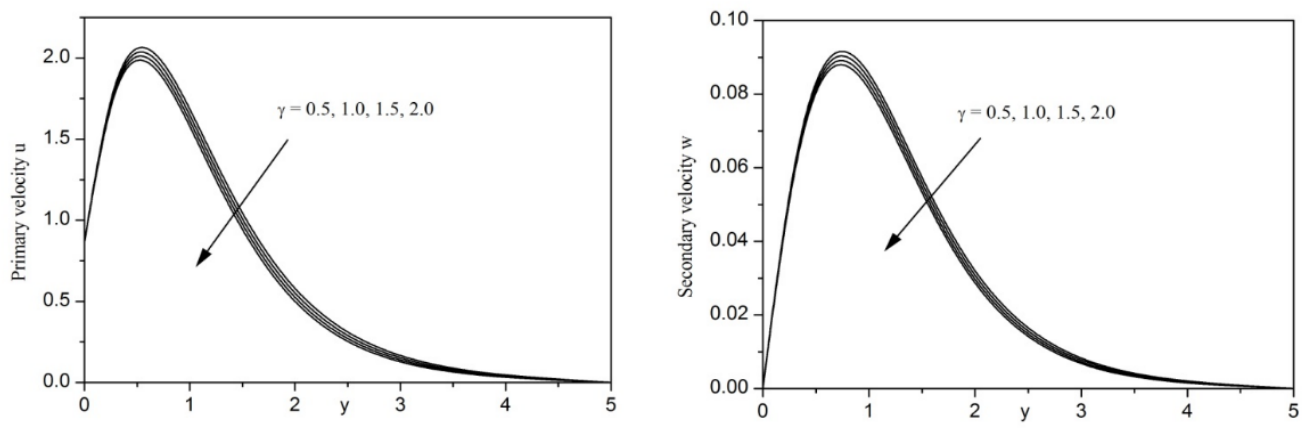

Fig. 8. Primary and secondary fluid velocities for varying chemical reaction parameter $\gamma$. 

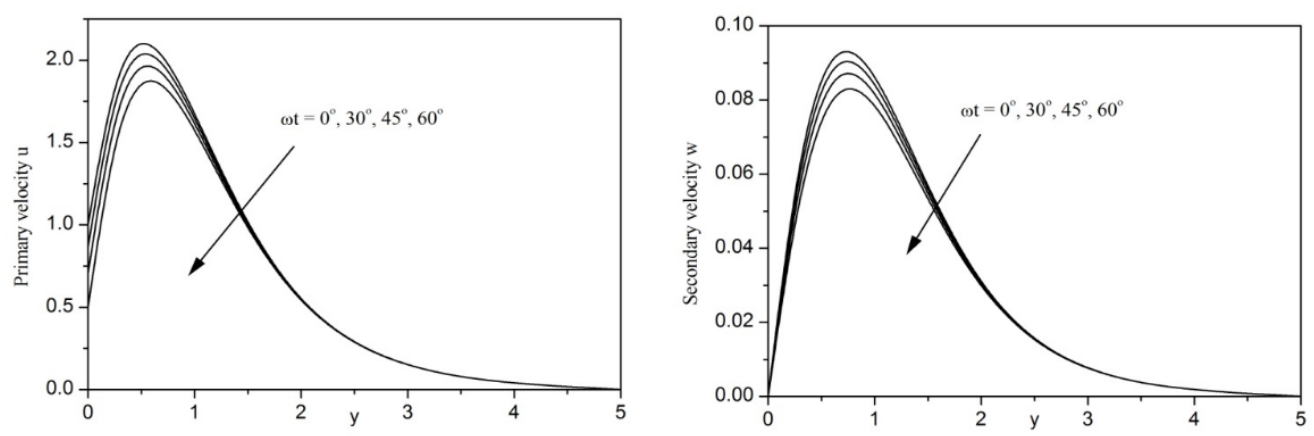

Fig. 9. Primary and secondary fluid velocities for varying phase angle $\omega t$.
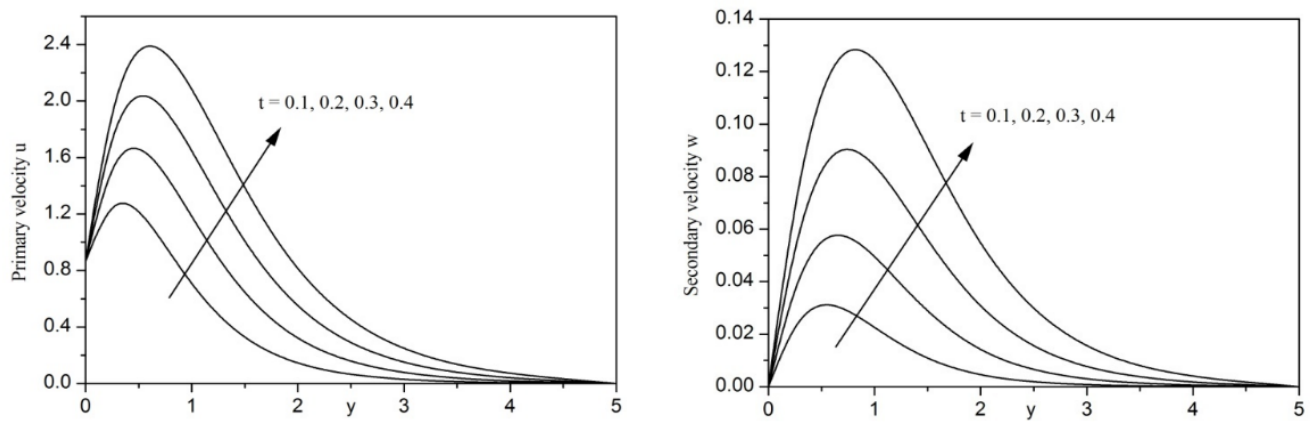

Fig. 10. Primary and secondary fluid velocities for varying time parameter $t$.

Figure 11 illustrates the effect of Prandtl number $P_{r}$ on the fluid temperature $\theta$. Increase in the Prandtl number corresponds to stronger momentum diffusivity and weaker diffusivity. This implies that increase in the Prandtl number tends to decrease the fluid temperature. The effect of time $t$ on the fluid temperature is shown in Fig. 12. It is clearly seen that as time progresses, the fluid temperature $\theta$ increases. 


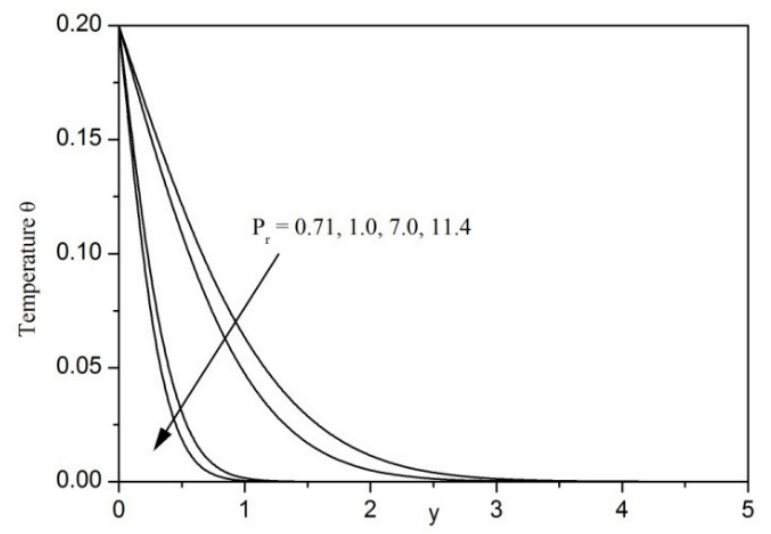

Fig. 11. Temperature $\theta$ for varying Prandtl number $P_{r}$.

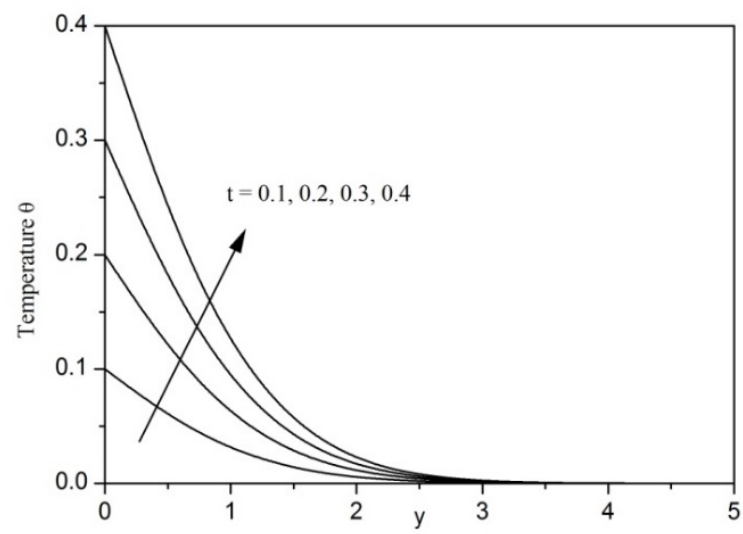

Fig. 12. Temperature $\theta$ for varying time parameter $t$.

The variation of fluid concentration $\phi$ for different values of Schmidt number $S_{c}$ is presented in Fig. 13. To be more realistic, the values of Schmidt number are chosen to represent the diffusing chemical species of most common interest like hydrogen $\left(S_{c}=0.23\right)$, water vapor $\left(S_{c}=0.64\right)$, sulfur dioxide $\left(S_{c}=1.20\right)$ and naphthalene $\left(S_{c}=2.23\right)$. It is noticed that an increase in Schmidt number tends to decrease the fluid concentration. Physically, increase in $S_{c}$ leads to decrease in molecular diffusivity which results in a decrease of concentration boundary layer. Figure 14 presents the effect of chemical reaction parameter $\gamma$ on the fluid concentration $\phi$. It can be seen that an increase in $\gamma$ tends to decrease concentration of species in the boundary layer since large values of $\gamma$ reduce the solutal boundary layer thickness and increase the mass transfer. The evolution of concentration $\phi$ with progression of time $t$ is depicted in Fig. 15. It is clear that fluid concentration increases with increasing time $t$. 


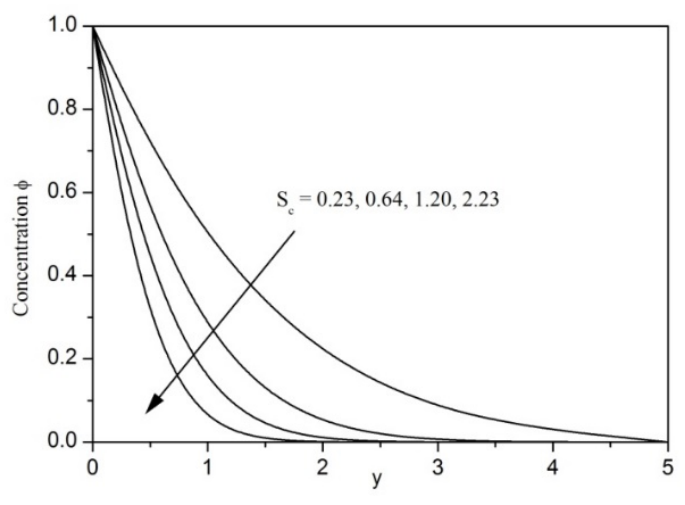

Fig. 13. Concentration $\phi$ for varying Schmidt number $S_{c}$.

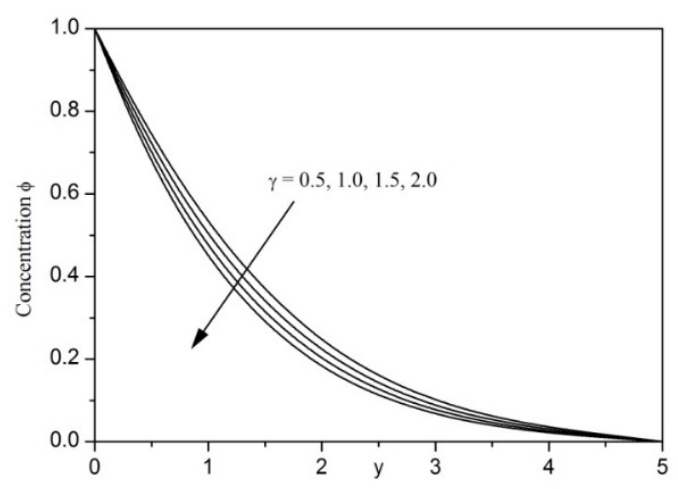

Fig. 14. Concentration $\phi$ for varying chemical reaction parameter $\gamma$.

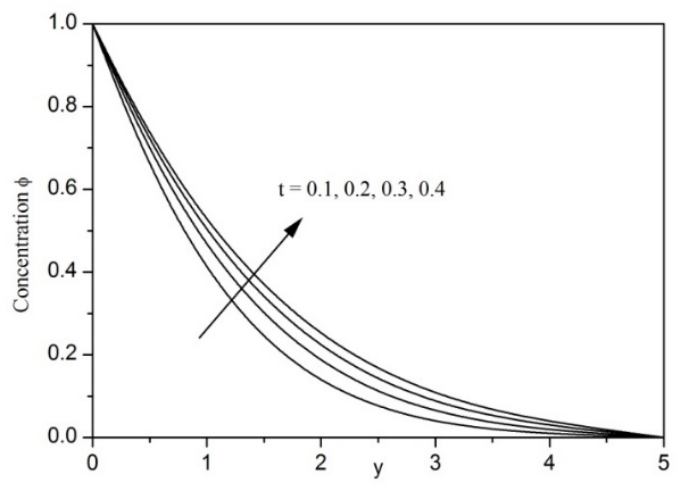

Fig. 15. Concentration $\phi$ for varying time parameter $t$. 
Table 3 presents the numerical values of Nusselt number for various values of Prandtl number and time parameter. As seen from Table 3, Nusselt number increases with increasing Prandtl number and time parameter. The numerical values of Sherwood number for various values of Schmidt number, chemical reaction parameter and time presented in Table 4. It is clearly seen from Table 4 that an increase in Schmidt number and chemical reaction tend to increase the Sherwood number whereas it decreases with increasing time.

\begin{tabular}{|c|c|c|}
\hline$P_{r}$ & $t$ & $N u$ \\
\hline 0.71 & 0.2 & 0.083336 \\
\hline 1.00 & 0.2 & 0.098578 \\
\hline 7.00 & 0.2 & 0.237110 \\
\hline 0.71 & 0.3 & 0.125004 \\
\hline 0.71 & 0.4 & 0.166670 \\
\hline
\end{tabular}

Table 3. Nusselt number for different values of $P_{r}$ and $t$

\begin{tabular}{|c|c|c|c|}
\hline$S_{c}$ & $\gamma$ & $t$ & $S h$ \\
\hline 0.23 & 1.0 & 0.2 & 0.296856 \\
\hline 0.64 & 1.0 & 0.2 & 0.481744 \\
\hline 0.23 & 2.0 & 0.2 & 0.349644 \\
\hline 0.23 & 1.0 & 0.3 & 0.280462 \\
\hline
\end{tabular}

Table 4. Sherwood number for different values of $S_{c}, \gamma$ and $t$.

\section{Conclusions}

The governing system of partial differential equations has been examined for the effects of chemical reaction on unsteady MHD flow past an impulsively started oscillating infinite vertical plate in the presence of Hall current. Significant findings of the study are summarized as follows:

1. Prandtl number, Schmidt number, chemical reaction parameter and phase angle tend to decelerate both primary and secondary fluid velocities whereas thermal Grashof number, mass Grashof number, Hall current and time tend to accelerate both primary and secondary velocities.

2. Magnetic parameter tends to retard the primary fluid velocity and opposite effect is observed on the secondary fluid velocity.

3. Prandtl number tends to decrease fluid temperature whereas time has the opposite effect.

4. Schmidt number and chemical reaction parameter tend to decrease the fluid concentration whereas the time has the opposite effect.

5. Primary and secondary skin frictions get reduced when Prandtl number and Schmidt number increase whereas the opposite effect is observed when thermal Grashof number, mass Grashof number, Hall current and time increase.

6. Magnetic parameter tends to decrease the primary skin friction whereas the reverse effect is observed on the secondary skin friction.

7. Phase angle tends to increase the primary skin friction whereas the opposite effect is observed on the secondary skin friction.

8. Nusselt number increases with increasing Prandtl number and time parameter. 
9. Schmidt number and chemical reaction parameter tends to increase Sherwood number and the opposite effect is observed when time progresses.

\section{Acknowledgements}

The authors wish to express their sincere gratitude to the editor and reviewers for careful reading of the manuscript and their valuable suggestions.

\section{References}

Anjalidevi S P, Kandasamy R (2000). Effects of chemical reaction, heat and mass transfer on MHD flow past a semi-infinite plate. Z. Angew. Math. Mech.. 80: 697-701.

Aboeldahab E M, Elbarbury E M E (2001). Hall current effect on magneto-hydrodynamic free convection flow past a semi-infinite vertical plate with mass transfer. Int. J. Eng. Sci. 39: $1641-1652$.

Acharya M, Dash G C, Singh L P (2001). Hall effect with simultaneous thermal and mass diffusion on unsteady hydro-magnetic flow near an accelerated vertical plate. Ind. J. Phys. 75B(1): $168-176$.

Cowling T C (1957). Magneto-hydrodynamics, Wiley Inter Science, New York.

Cortell R (2007). MHD flow and mass transfer of an electrically conducting fluid of second grade in a porous medium over stretching sheet with chemically reactive species, Chem. Eng. Process. 46: $721-728$.

Das U N, Deka R K, Soundalgekar V M (1994). Effects of mass transfer on flow past an impulsively started infinite vertical plate with constant heat flux and chemical reaction, Forsch Ingenieurwes. 60: 284-287.

Davidson P A (1999). Magneto-hydrodynamics in material processing - Annual Review Fluid Mech. 31: 273-300.

El-Fayez F M N (2012). Effects of chemical reaction on the unsteady free convection flow past an infinite vertical permeable moving plate with variable temperature. J. Surface Engineered Materials and Adv. Tech.. 2: 100-109.

Fife J (1998). Hybrid-PIC Modeling and electrostatic probe survey of Hall thrusters - $\mathrm{PhD}$ Thesis, Department of Aeronautics and Astronautics, MIT, USA.

Hardianto T, Sakamoto N, Harada N (2008). Computational study of diagonal channel magnetohydrodynamic power generation. Int. J. Energy Tech. Policy. 6: 96-111.

Kholshchevnikova E K (1966). Influence of the Hall effect on the characteristics of a MHD generator with two pairs of electrodes. J. Appl. Mech. Tech. Phys. 7(4): 48-54.

Muthucumaraswamy R, Ganesan P (2001). First order chemical reaction on flow past an impulsively started vertical plate with uniform heat and mass flux. Acta Mechanica. 147: 4557.

Morley N B, Malang S, Kirillov I (2005). Thermo-fluid Magneto-hydrodynamic issues for liquid breeders - Fission Sci. Tech. 47: 488-501.

Muthucumaraswamy R, Meenakshisundaram S (2006). Theoretical study of chemical reaction effects on vertical oscillating plate with variable temperature. Theo. Appl. Mech. 33(3): 245257.

Muthucumaraswamy R, Janakiraman B (2008). Mass transfer effects on isothermal vertical oscillating plate in the presence of chemical reaction. Int. J. Appl. Math and Mech. 4(1): 6674. 
Mathon Ph, Nouri A, Alemany A, Chopart J P, Sobolik V, Baaziz D (2009). Electro-chemical processes controlled by high magnetic fields: applications to MHD sea water propulsion Magneto-hydrodynamics. 45: 281-288.

Mahapatra N, Dash G C, Panda S, Acharya M (2010). Effects of chemical reaction on free convection flow through a porous medium bounded by a vertical surface. J. Eng. Phys and Thermo-Phys. 83(1): 130-140.

Michaeli K, Tikhonov K S, Finkel'stein A M (2012). Hall effect in super conducting films Physical Review. B 86: 014515.

Maguna A N, Mutua N M (2013). Hall current effects on free convection flow and mass transfer past a semi infinite vertical flat plate. Int. J. Mathematics and Statistics studies. 1(4): 1-22.

Pop I (1971). The effect of Hall current on hydro-magnetic flow near an accelerated plate. J. Math. Phys. Sci. 5: 375-379.

Prabhakar Reddy B (2018). Hall effect on MHD transient flow past an impulsively started infinite horizontal porous plate in a rotating system. Int. J. Appl. Mech and Eng. 23(2): $471-483$.

Raptis A, Perdikis C (2006). Viscous flow over a non-linearly stretching sheet in the presence of chemical reaction and magnetic field. Int. J. Non-Linear Mech. 41: 527-529.

Rajesh V, Varma S V K (2010). Chemical reaction effects on free convection flow past an exponentially accelerated vertical plate. Annals. J. Eng., VIII, Fascicule 1: $181-188$.

Rajesh V (2010). MHD and chemical reaction effects on free convection flow with variable temperature and mass diffusion. Annals. J. Eng., VIII, Fascicule 3: 370 - 378.

Rajput U S, Neetu Kanaujia (2016). MHD flow past a vertical plate with variable temperature and mass diffusion in the presence of Hall current, Int. J. Appl. Sci. and Eng. 14(2): 115 123.

Shang J S, Surzhikov S T, Kimmel R, Gaitonde D, Menart J, Hayes J (2005). Mechanics of plasma actuators for hypersonic flow control - Prog. Aerospace. Sci., 41, 642-668.

Sharma B K, Jha A K, Chaudary R C (2007). Hall effect on MHD mixed convection flow of a viscous incompressible fluid past a vertical porous plate immersed in a porous medium with heat source/sink, Rom. J. Phys., 52(5-7): 487 - 503.

Sehkar D V, Reddy G V (2012). Effects of chemical reaction on MHD free convective oscillatory flow past a porous plate with viscous dissipation and heat sink. Adv. Appl. Sci. Res. 3(5): 3206-3215.

Thamizhsudar M, Pandurangan J, Muthucumaraswamy R (2015): Hall effects and rotation effects on MHD flow past an exponentially accelerated vertical plate with combined heat and mass transfer effects. Int. J. Appl. Mech and Eng. 20, 3: 605-616.

Van Wie D M. (2005). Future Technologies - Application of Plasma Devices for Vehicle System - The Johas Hopkins University, Applied Physics Laboratory - Laurel, Maryland, USA NATO Document.

Watanabe T, Pop I (1995). Hall effect on magneto-hydrodynamic boundary layer flow over a continuous moving flat plate. Acta Mechanica. 108: 35-47. 